\title{
Diferencias de la felicidad según sexo y edad en universitarios peruanos
}

\author{
Happiness Differences by Sex and Age in Peruvian University
}

\author{
Humberto Castilla ${ }^{1}$ \\ Tomás Caycho ${ }^{2}$ \\ José Luís Ventura-León ${ }^{3}$ \\ Universidad Privada del Norte, Perú
}

Resumen. El estudio, descriptivo comparativo, explora las diferencias de la felicidad en jóvenes y adultos peruanos, según el sexo y la edad, a partir de las puntuaciones de la Escala de Felicidad de Lima (Alarcón, 2006). Para ello se contó con la participación de 402 personas, varones (50\%) y mujeres (50\%), con edades entre 16 y 35 años (Media = 19.59; DT = 3.75) de la ciudad de Lima. Los resultados muestran que las mujeres son más propensas a experimentar cierto grado de felicidad; mientras que las personas del grupo de mayor edad tienen más altas puntuaciones en felicidad que las del grupo de menor edad. Los resultados contribuyen a una mejor comprensión de la felicidad y su relación con variables sociodemográficas vinculadas a la salud y el bienestar. Esto permite, a su vez, contar con información empírica importante para la implementación de acciones destinadas a favorecer el desarrollo de variables positivas.

Palabras clave. Edad, felicidad, psicología positiva, sexo.

Abstract. The study, comparative descriptive, describes the differences of happiness in Peruvian youth and adults based on sex and age using the Lima Happiness Scale (Alarcon, 2006). 402 people, male (50 \%) and women (50 \%), between 16 and 35 years old $($ mean $=19.59, \mathrm{SD}=3.75)$ of the city of Lima participated in the study. The results show that women are more likely to experience some degree of happiness; while people of the older age group have higher happiness scores than the younger age group. The results contribute to a better understanding of happiness and its relation to sociodemographic variables related to health and well-being. This provides important empirical information for the implementation of actions to promote the development of positive variables

Keywords. Age, happiness, positive psychology, sex.

${ }^{1}$ Humberto Castilla Cabello. Universidad Privada del Norte, Perú. E-mail: humberto.castilla@upn.pe

${ }^{2}$ Tomás Caycho. Universidad Privada del Norte, Perú. Dirección postal: Av. Tingo María 1122, Cercado, Lima, Perú. E-mail: tomas.caycho@upn.pe J3osé Luís Ventura-León. Universidad Privada del Norte, Perú. E-mail: jose.ventura@upn.pe

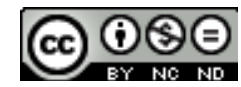

Esta obra está bajo una licencia de Creative Commons Reconocimiento-NoComercial-SinObraDerivada 4.0 Internacional. 


\section{Introducción}

En la actualidad, la psicología positiva se encuentra en pleno desarrollo y centra su interés en el estudio de las cualidades y emociones positivas de las personas, con el objetivo de mejorar su calidad de vida (Seligman \& Csikszentmihalyi, 2000). Entre la gran cantidad de temas abordados por este enfoque, está el estudio de la felicidad, que ha generado enorme interés de la comunidad científica psicológica; hasta el año 2010, este presentaba el mayor número de publicaciones en comparación con otros temas dentro de este enfoque psicológico (Caycho, 2010).

Con respecto a la conceptualización de la felicidad, esta ha sido estudiada desde varios dominios como la filosofía. En el presente, viene siendo tema de estudio de la psicología desde una perspectiva científica. En esta línea, Alarcón (2009) refiere que, dentro de los estudios teóricos, la conceptualización de la felicidad ha recibido un notable interés. Para algunos autores (Diener, 1984; Csickszentmihalyi, 2008; Cuadra \& Florenzano, 2003; Lyubomirsky, 2008), la felicidad ha sido definida como un proceso de evaluación general y positiva que realiza una persona en un momento específico, que permite controlar cognitivamente su percepción de sí misma y del contexto que la rodea.

Asimismo, en la literatura científica, algunos trabajos equiparan la felicidad con un estado de bienestar subjetivo (Diener, Emmons, Larsen \& Griffin, 1985; Diener, Suh, Lucas \& Smith, 1999) y satisfacción hacia la vida (Vennhoven, 2005; Frey \& Stutzer, 2000). Según Michalos (1995a, 1995b), tanto la felicidad, el bienestar subjetivo y la satisfacción posen similar significado, lo que lleva a considerar la teoría de la satisfacción con la vida como una teoría de la felicidad; ambas dentro de las teorías del bienestar subjetivo. Tratando de aportar la claridad a esta discusión, Alarcón (2006) señala que, tanto la felicidad como el bienestar subjetivo, pueden ser utilizados como sinónimos, con el propósito de facilitar el análisis científico de esta variable; señalando, sin embargo, que por sí mismo, el término felicidad explicaría un estado afectivo.
En este sentido, un análisis psicológico de la felicidad permite definirla como un "estado afectivo de satisfacción plena que experimenta subjetivamente el individuo en posesión de un bien anhelado" (Alarcón, 2006, p. 101). Este se puede expresar cuantitativamente, siendo factible su medición en términos de grados de satisfacción.

La definición anteriorpermite tomarenconsideración algunos indicadores importantes. Primero, la felicidad hace referencia a una satisfacción experimentada subjetivamente por una persona y solo por ella. En segundo lugar, considerar a la felicidad como un estado hace referencia a su dimensión temporal. Finalmente, un tercer indicador, relaciona el logro de la felicidad con la posesión de bienes u objetos que puede ser de diversa naturaleza; por ejemplo, bienes psicológicos, religiosos, sociales, entre otros (Alarcón, 2006).

En función de esta caracterización de la felicidad, Alarcón (2006), señala cuatro dimensiones que la subyacen. Primero, se menciona la ausencia de sufrimiento profundo, que expresa un conjunto de actitudes positivas hacia sí mismo y la vida, alejada de estados depresivos profundos. La segunda dimensión es denominada satisfacción con la vida y hace referencia al grado de gozo que la persona ha alcanzado en relación con lo que tiene o se encuentra cerca de conseguir. La tercera dimensión, realización personal, evidencia la felicidad plena, contraria a estados temporales de felicidad. Asimismo, guarda correspondencia con la orientación hacia metas que la persona considera valiosas para su vida. Finalmente, la dimensión alegría de vivir, expresa el optimismo y alegría, espera de que el futuro depare resultados favorables, y ayuda a enfrentar los problemas con una actitud positiva (Alarcón, 2006, 2009).

Por otra parte, la importancia del estudio de la felicidad se expresa por su relación con variables asociadas a los procesos de salud. La felicidad se encuentra relacionada con la capacidad de perdonar (Makinen \& Jhonson, 2006), la esperanza (Bailey, Eng, Frisch \& Snyder, 2007; Halama, 2010; Chen \& Chen, 2008), el optimismo (Salgado, 2009) y la gratitud (Bono, Emmons \& McCulluogh, 2004; Emmons, 
2008; Emmons \& McCulluogh, 2003; Macnulty, 2004), siendo la variable reciprocidad uno de los mejores predictores de la felicidad (Alarcón \& Caycho, 2015). Por su parte, Alarcón (2009) ha hallado que la felicidad se relaciona significativamente con variables familiares, la salud, religión, así como el altruismo y la bondad (Seligman, 2006; Niven, 2011).

En relación con la existencia de diferencias significativas de la felicidad entre hombres y mujeres, los resultados son contradictorios. Algunos estudios de meta-análisis sugieren que las mujeres se encuentran más proclives a experimentar estados de felicidad a diferencia de los hombres (Francis, 1999; Lu, 1996; Mookherjee, 1997; Pavot, Diener \& Fugita, 1990; Seligman, 2011; Wood, Rhodes \& Whelan, 1989), aunque se han reportado, también, investigaciones con resultados contrarios que mencionan que el sexo no es suficiente para la explicación de la existencia de diferentes niveles de felicidad (Alarcón, 2001; Alarcón \& Caycho, 2015; Argyle, 1990; Diener \& Diswas, 2008; Laca, Verdugo \& Guzman, 2005; Seligman, 2011).

En cuanto a las diferencias de la felicidad en función de la edad, Alarcón (2001), señala que no se presentan diferencias, y menciona que la única diferencia importante se da entre los 30 y 50 años. El mismo autor señala, además, que los niveles más elevados de felicidad corresponden a edades entre 50 y 60 años de edad. En un estudio (Mroczek \& Kolarz, 1998) realizado con 2727 hombres y mujeres entre los 25 y 74 años edad, se señaló que, a mayor edad, las personas reportaron ser más felices y con menos presencia de emociones negativas.

En el caso del grupo de adultos jóvenes, estos reportaron una mayor frecuencia de emociones negativas y sentimientos de tristeza, (Mroczek \& Kolarz, 1998). En el mismo sentido, otras investigaciones señalan que la satisfacción con la vida no muestra disminución conforme se avanza en edad; mientras que los afectos positivos sí presentan un descenso entre los grupos de edad (Diener \& Suh, 1998).

De los hallazgos anteriores para el sexo, la edad y la felicidad se puede afirmar que las variables mediadoras involucradas en su relación pueden ser diversas. Así, algunos estudios concuerdan en que la mayoría de personas asegura tener niveles moderados de felicidad, con independencia de su edad y sexo (Myers, 2000). Otros señalan justificable estudiar el tema porque las variables pueden estar mediando en los resultados obtenidos, tales como el empleo de muestras no representativas del contexto en que se llevan a cabo, el papel de efecto diferencial del sexo en diversos periodos del ciclo vital y la participación del estado civil y la situación laboral y la edad (Easterlin, 2003).

Así, si hombres y mujeres presentan cambios similares a lo largo del ciclo vital conforme se avanza en edad, es de esperar que la felicidad varíe de manera similar; sin embargo, se observan cambios diferentes (Easterlin, 2003). En el caso de la edad, algunos estudios señalan que los niveles de felicidad aumentan a lo largo del ciclo de vida (Mroczek \& Kolarz, 1998); otros, mencionan la existencia de una relación en forma de $\mathrm{U}$, donde el punto más alto se encuentra en torno a los 40 años (Blanchflower \& Oswald, 2000). Por último, algunos estudios no reportan ninguna tendencia en torno a la felicidad (Costa et al., 1987; Myers \& Diener, 1996).

En el Perú, pocos estudios analizan la felicidad y su relación con otras variables sociodemográficas como el sexo, la edad y estado conyugal (Alarcón, 2001; Alarcón \& Caycho, 2015). Con base en la evidencia teórica y empírica mostrada, se expresa una limitada cantidad de investigaciones sobre la felicidad en jóvenes y adultos peruanos, así como resultados poco concluyentes de las investigaciones previas, siendo necesario generar información empírica para tener una mejor comprensión de cómo se relaciona la felicidad y las variables arriba mencionadas. Estas evidencias sugieren la relevancia de analizar las diferencias de la felicidad en jóvenes y adultos universitarios peruanos en relación con sexo y la edad.

\section{Método}

El estudio es de corte transversal (Montero \& León, 2007) de alcance descriptivo comparativo (Hernández, Fernández \& Baptista, 2010). 


\section{Participantes}

El estudio se realizó con la participación de 402 personas, en donde el $50 \%$ son varones y el $50 \%$ restante son mujeres. Cursaban estudios en una universidad privada de Lima (Perú), y sus edades oscilaban entre 16 y 35 años, con una edad promedio de 19.59 años $(\mathrm{DT}=3.751)$. Los participantes fueron seleccionados a través de un muestreo no probabilístico por conveniencia, con base en los siguientes criterios de inclusión: a) residir en la ciudad de Lima; b) haber nacido en el Perú; c) pertenecer a una universidad particular y d) que fueran de ambos sexos.

\section{Instrumentos}

Escala de Felicidad de Lima (EFL). Desarrollada por Alarcón (2006), es una escala conformada por 27 ítems con cinco alternativas de respuesta tipo Likert, distribuidos de forma aleatoria y redactados en un sentido favorable o desfavorable hacia la felicidad, donde el extremo positivo (totalmente de acuerdo) recibe un valor de cinco puntos y al extremo negativo un punto (totalmente en desacuerdo). La EFL fue administrada a 709 personas, hombres y mujeres con un promedio de 23.1 años. Evalúa cuatro dimensiones, las cuales presentan altas cargas factoriales $(.413 \mathrm{a}$ $.689)$ : ausencia de sufrimiento profundo (11 ítems; $\alpha$ $=0.88$ ), satisfacción con la vida (6 ítems; $\alpha=.79$ ), realización personal ( 6 ítems; $\alpha=.76$ ) y alegría de vivir (4 ítems; $\alpha=.72$ ).

\section{Procedimiento}

Primero, se solicitaron los permisos a las autoridades de la universidad seleccionada para la evaluación, dándole información sobre el objetivo de la investigación y las características del instrumento a aplicar. Luego de obtenidos el permiso y la colaboración de las autoridades universitarias, se procedió a informar a los estudiantes el objetivo del estudio, contando con su participación voluntaria, se firmanel consentimiento informado, garantizando así la confiabilidad y el anonimato de la información personal suministrada. Se realizó la aplicación colectiva

Tabla 1

Características sociodemográficas de la muestra $(\mathrm{N}=402)$

\begin{tabular}{lcc}
\hline & Frecuencia & Porcentaje \\
\hline Sexo & & \\
$\quad$ Varón & 201 & $50 \%$ \\
$\quad$ Mujer & 201 & $50 \%$ \\
Edad & 212 & $52.7 \%$ \\
$\quad$ Joven (16 a 18 años) & 190 & $47.3 \%$ \\
$\quad$ Adulto (19 a 35 años) & & \\
Ciclo académico & 275 & $68.4 \%$ \\
1er y 2do año & 127 & $31.6 \%$ \\
3er y 4to año & & \\
Estrato socioeconómico & 22 & $5.5 \%$ \\
$\quad$ Alto & 363 & $99.3 \%$ \\
Medio & 17 & $4.2 \%$ \\
$\quad$ Bajo & & \\
Ocupación & 58 & $14.4 \%$ \\
$\quad$ Estudia & 344 & $100 \%$ \\
$\quad$ Trabaja y estudia & 420 & \\
Total & &
\end{tabular}


de la EFL, contando para ello de la colaboración de un conjunto de asistentes de investigación, que fue previamente capacitado en la aplicación y corrección de la EFL.

Para el análisis comparativo se utilizó la prueba U de Mann-Whitney y la probabilidad de superioridad (PS) como indicador del tamaño del efecto, presentando las siguientes categorías interpretativas: No efecto, (PSest $\leq$ $=.50)$ pequeño (PSest $\geq .56$ ), mediano (PSest $\geq$.64) y grande (PSest $\geq$.71) (Erceg-Hurn \& Mirosevich, 2008; Grissom, 1994). Los datos obtenidos se analizaron por medio del paquete estadístico SPSS versión 22.

\section{Resultados}

\section{Análisis descriptivo}

Primero, se analizó distribución normal de los datos, con el fin de elegir apropiadamente los estadísticos. Se utilizó la prueba de bondad de ajuste KolmogorovSmirnov para cada una de las dimensiones de la EFL. Los hallazgos indican que todas las dimensiones de la escala siguen una distribución no paramétrica (véase tabla 2), por tal razón, se utilizó para el análisis de las puntuaciones la prueba U de Mann-Whitney y la probabilidad de superioridad (PS) (Erceg-Hurn \& Mirosevich, 2008; Grissom, 1994) como indicador del tamaño del efecto.

\section{Diferencias en la felicidad en función al sexo}

En la tabla 3, se observa que, en promedio, las mujeres tienen más altas puntuaciones en la EFL $\left(\mathrm{Rp}_{\text {masculino }}=\right.$ 200.18; $\mathrm{Rp}_{\text {femenino }}=202.82$ ), siendo la magnitud de la diferencia considerada nula $\left(\mathrm{PS}_{\text {est }}=.49\right)$. La diferencia más notable ocurre en la dimensión sentido positivo de la vida $\left(\mathrm{Rp}_{\text {masculino }}=200.74 ; \mathrm{Rp}_{\text {femenino }}=202.26, \mathrm{PS}_{\text {est }}\right.$ $=.49$ ), seguido de la dimensión realización personal $\left(\mathrm{Rp}_{\text {masculino }}=196.76 ; \mathrm{Rp}_{\text {femenino }}=206.24, \mathrm{PS}_{\text {est }}=.47\right)$ pero también contando con una diferencia nula. Asimismo, se evidencia que los varones presentan puntuaciones altas en la dimensión satisfacción con la vida $\left(\mathrm{Rp}_{\text {masculino }}=202.69 ; \mathrm{Rp}_{\text {femenino }}=200.69, \mathrm{PS}_{\text {est }}=\right.$ .49) y alegría de vivir $\left(\mathrm{Rp}_{\text {masculino }}=202.99 ; \mathrm{RP}_{\text {femenino }}=\right.$ 200.01, PS est $=.49)$, con una magnitud cercana a ser considerada pequeña.

En relación con la primera dimensión, sentido positivo de la vida, se observan diferencias cercanas a ser consideradas moderadas a favor de los varones en el ítem 11 (La mayoría del tiempo me siento feliz*) $\left(\mathrm{Rp}_{\text {masculino }}=203.54 ; \mathrm{Rp}_{\text {femenino }}=199.46, \mathrm{PS}_{\text {est }}=.49\right)$; y a favor de las mujeres en el ítem 20 (La felicidad no es para algunas personas, sino también para mí) $\left(\mathrm{Rp}_{\text {masculino }}=198.98 ; \mathrm{Rp}_{\text {femenino }}=204.02, \mathrm{PS}_{\text {est }}=.49\right) \mathrm{y} \mathrm{el}$ ítem 22 (Me siento alegre por lo que soy*) $\left(\mathrm{Rp}_{\text {masculino }}=\right.$ 199.33; $\mathrm{Rp}_{\text {femenino }}=203.67, \mathrm{PS}_{\text {est }}=.49$ ). En la dimensión satisfacción con la vida, se evidencia una diferencia a favor de los varones en el reactivo 4 (Estoy satisfecho con mi vida) $\left(\mathrm{Rp}_{\text {masculino }}=202.83 ; \mathrm{Rp}_{\text {femenino }}=200.17\right.$, $\left.\mathrm{PS}_{\text {est }}=.49\right)$ y la diferencia más pequeña en el reactivo 6 (Me siento satisfecho con lo que soy) $\left(\mathrm{Rp}_{\text {masculino }}=\right.$ 208.53; $\mathrm{Rp}_{\text {femenino }}=194.47, \mathrm{PS}_{\text {est }}=.46$ ).

Por otra parte, en la dimensión realización personal, se observan diferencias a favor de las mujeres en el ítem 24 (Me considero una persona realizada) $\left(\mathrm{Rp}_{\text {masculino }}=\right.$ 200.15; $\mathrm{Rp}_{\text {femenino }}=202.85, \mathrm{PS}_{\text {est }}=.49$ ), ítem 25 (Mi vida transcurre plácidamente) $\left(\mathrm{Rp}_{\text {masculino }}=200.84 ; \mathrm{Rp}_{\text {femenino }}\right.$

Tabla 2

Análisis de la Bondad de Ajuste a la curva normal de las variables en estudio en la muestra total $(N=402)$

\begin{tabular}{lcccc}
\hline \multicolumn{1}{c}{ Variables } & $M$ & D.T. & K-S & Sig. \\
\hline Sentido positivo de la vida & 42.71 & 7.77 & .101 & .0001 \\
Satisfacción con la vida & 21.85 & 3.84 & .110 & .0001 \\
Realización personal & 19.69 & 4.09 & .062 & .001 \\
Alegría de vivir & 15.74 & 2.84 & .141 & .0001 \\
Escala total & 100 & 15.379 & .076 & .0001 \\
\hline
\end{tabular}


Tabla 3

Rangos promedio de las dimensiones de la escala de felicidad de lima de acuerdo al sexo

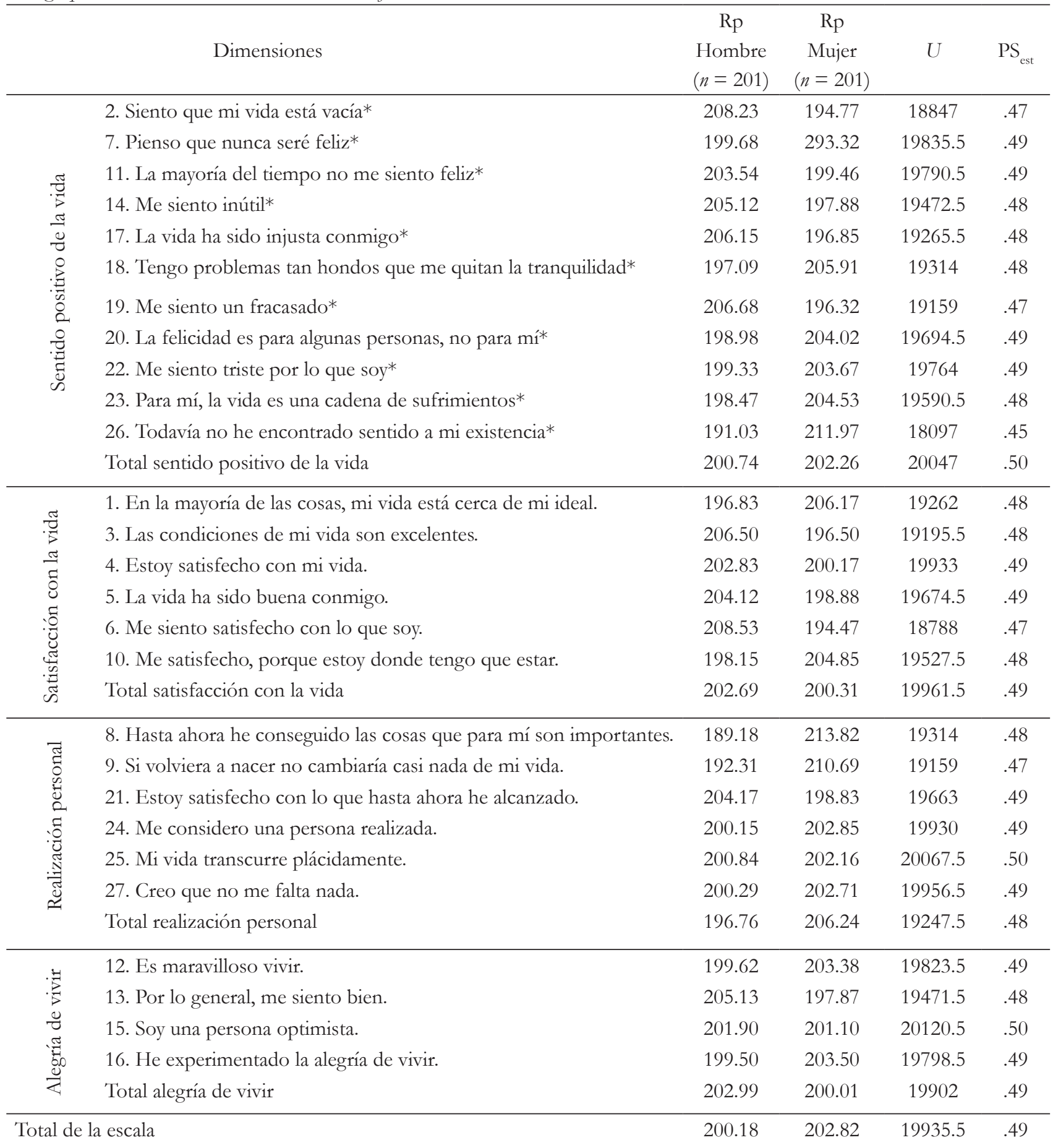

Nota. * Ítems invertidos; PS : probabilidad de superioridad (tamaño del efecto); Rp: rangos promedios; U: Mann-Whitney. 
$=202.16, \mathrm{PS}_{\text {est }}=.49$ ) y el ítem 27 (Creo que no me falta nada) ( $\mathrm{Rp}_{\text {masculino }}=200.29 ; \mathrm{Rp}_{\text {femenino }}=202.71$, $\left.\mathrm{PS}_{\text {est }}=.49\right)$. Finalmente, en la dimensión alegría de vivir, se observan diferencias consideradas a favor de los varones en el ítem 15 (Soy una persona optimista) $\left(\mathrm{Rp}_{\text {masculino }}=201.9 ; \mathrm{Rp}_{\text {femenino }}=201.1, \mathrm{PS}_{\text {est }}=.49\right) \mathrm{y} \mathrm{a}$ favor de las mujeres en el ítem 12 (Es maravilloso vivir) $\left(\mathrm{Rp}_{\text {masculino }}=199.62 .13 ; \mathrm{Rp}_{\text {femenino }}=203.38, \mathrm{PS}_{\text {est }}=.49\right)$ y el ítem 16 (He experimentado la alegría de vivir) $\left(\mathrm{Rp}_{\text {masculino }}=199.5 ; \mathrm{Rp}_{\text {femenino }}=203.5, \mathrm{PS}_{\text {est }}=.49\right)$.

\section{Diferencias en la felicidad en función a la edad}

En la tabla 4 se observa que, en promedio, los adultos tienen más altas puntuaciones en la Escala de Felicidad de Lima $\left(\mathrm{Rp}_{\text {ióvenes }}=183.23 ; \mathrm{RP}_{\text {adultos }}=221.89\right)$, no presentando magnitud de la diferencia $\left(\mathrm{PS}_{\mathrm{est}}=.40\right)$. La diferencia más notable ocurre en la dimensión satisfacción con la vida $\left(\mathrm{Rp}_{\text {jóvenes }}=194.84 ; \mathrm{Rp}_{\text {adultos }}=208.93, \mathrm{PS}_{\text {est }}=.46\right)$, seguido de la dimensión realización personal $\left(\mathrm{Rp}_{\text {ióvenes }}=196.76\right.$; $\mathrm{Rp}_{\text {adultos }}=206.24, \mathrm{PS}_{\text {est }}=.45$ ), donde la magnitud de la diferencia está cercana a ser considerada pequeña.

Tabla 4

Rangos promedio de las dimensiones de la Escala de Felicidad de Lima de acuerdo a la edad

\begin{tabular}{|c|c|c|c|c|c|}
\hline & Dimensiones & $\begin{array}{c}\mathrm{Rp} \\
\text { Jóvenes } \\
(n=212)\end{array}$ & $\begin{array}{c}\mathrm{Rp} \\
\text { Adultos } \\
(n=190)\end{array}$ & $U$ & $\mathrm{PS}_{\text {est }}$ \\
\hline \multirow{12}{*}{ 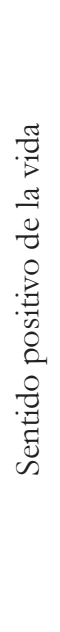 } & 2. Siento que mi vida está vacía* & 187.04 & 217.63 & 17075.5 & .42 \\
\hline & 7. Pienso que nunca seré feliz* & 188.47 & 216.03 & 17378.5 & .43 \\
\hline & 11. La mayoría del tiempo no me siento feliz* & 197.67 & 205.78 & 19327 & .48 \\
\hline & 14. Me siento inútil* & 188.82 & 215.64 & 17452.5 & .43 \\
\hline & 17. La vida ha sido injusta conmigo* & 178.15 & 227.55 & 15190 & .38 \\
\hline & 18. Tengo problemas tan hondos que me quitan la tranquilidad* & 179.84 & 225.67 & 15548 & .39 \\
\hline & 19. Me siento un fracasado* & 191.13 & 213.07 & 17942 & .45 \\
\hline & 20. La felicidad es para algunas personas, no para mí* & 186.40 & 218.35 & 16938 & .42 \\
\hline & 22. Me siento triste por lo que soy* & 196.33 & 207.27 & 19043 & .47 \\
\hline & 23. Para mí, la vida es una cadena de sufrimientos* & 177.56 & 228.22 & 15064 & .37 \\
\hline & 26. Todavía no he encontrado sentido a mi existencia* & 192.61 & 211.42 & 18255.5 & .45 \\
\hline & Total sentido positivo de la vida & 178.53 & 227.13 & 15270 & .38 \\
\hline \multirow{7}{*}{ 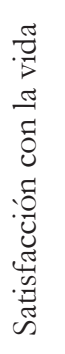 } & 1. En la mayoría de las cosas, mi vida está cerca de mi ideal. & 189.66 & 214.71 & 17630.5 & .44 \\
\hline & 3. Las condiciones de mi vida son excelentes. & 197.46 & 206.01 & 19282.5 & .48 \\
\hline & 4. Estoy satisfecho con mi vida. & 205.03 & 197.56 & 19391 & .48 \\
\hline & 5. La vida ha sido buena conmigo. & 193.10 & 210.87 & 18359.5 & .46 \\
\hline & 6. Me siento satisfecho con lo que soy. & 204.29 & 198.38 & 19547.5 & .49 \\
\hline & 10. Me satisfecho, porque estoy donde tengo que estar. & 194.91 & 208.86 & 18742.5 & .47 \\
\hline & Total satisfacción con la vida & 194.84 & 208.93 & 18728 & .46 \\
\hline
\end{tabular}

Continúa... 


\begin{tabular}{|c|c|c|c|c|c|}
\hline \multirow{7}{*}{ 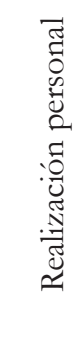 } & 8. Hasta ahora he conseguido las cosas que para mí son importantes. & 191.56 & 212.59 & 18033.5 & .45 \\
\hline & 9. Si volviera a nacer no cambiaría casi nada de mi vida. & 192.29 & 211.77 & 18188.5 & .45 \\
\hline & 21. Estoy satisfecho con lo que hasta ahora he alcanzado. & 203.78 & 198.96 & 19657 & .49 \\
\hline & 24. Me considero una persona realizada. & 190.89 & 213.34 & 17891 & .44 \\
\hline & 25. Mi vida transcurre plácidamente. & 195.5 & 208.2 & 18867.5 & .47 \\
\hline & 27. Creo que no me falta nada. & 207.16 & 195.18 & 18940 & .47 \\
\hline & Total realización personal & 193.63 & 210.28 & 18472.5 & .46 \\
\hline \multirow{5}{*}{ 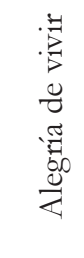 } & 12. Es maravilloso vivir. & 194.08 & 209.78 & 18567.5 & .46 \\
\hline & 13. Por lo general, me siento bien. & 187.96 & 216.61 & 17270 & .43 \\
\hline & 15. Soy una persona optimista. & 185.52 & 219.33 & 16751.5 & .42 \\
\hline & 16. He experimentado la alegría de vivir. & 188.07 & 216.49 & 17292 & .43 \\
\hline & Total alegría de vivir & 183.57 & 221.51 & 16338 & .41 \\
\hline \multicolumn{2}{|c|}{ Total de la escala } & 183.23 & 221.89 & 16266.5 & .40 \\
\hline
\end{tabular}

Nota. * Ítems invertidos; PS est probabilidad de superioridad (tamaño del efecto); Rp: rangos promedios; U: Mann-Whitney. Jóvenes $=16$ a 18 años; Adultos $=19$ a 35 años.

En relación con la primera dimensión, sentido positivo de la vida, se observan diferencias cercanas a ser consideradas moderadas a favor de los adultos en el ítem 11 (La mayoría del tiempo me siento feliz*) $\left(\mathrm{Rp}_{\text {ióvenes }}=197.67 ; \mathrm{Rp}_{\text {adultos }}=205.78, \mathrm{PS}_{\text {est }}=\right.$ .48) y la diferencia más pequeña se da en el ítem 17 (La vida ha sido justa conmigo*) $\left(\mathrm{Rp}_{\text {ióvenes }}=178.15\right.$; $\mathrm{Rp}_{\text {adultos }}=227.55, \mathrm{PS}_{\text {est }}=.37$ ) y el ítem 23 (Para mí, la vida no es una cadena de sufrimiento*) $\left(\mathrm{Rp}_{\text {ióvenes }}=\right.$ 177.56; $\left.\mathrm{Rp}_{\text {adultos }}=228.22, \mathrm{PS}_{\mathrm{est}}=.37\right)$. En la dimensión satisfacción con la vida, se evidencia una diferencia a ser considerada moderada a favor de los jóvenes en el reactivo 4 (Estoy satisfecho con mi vida) $\mathrm{Rp}_{\text {jóvenes }}$ $\left.=202.83 ; \mathrm{Rp}_{\text {adultos }}=200.17, \mathrm{PS}_{\text {est }}=.49\right)$ y la diferencia más pequeña a favor de los adultos en el reactivo 5 (la vida ha sido buena conmigo $)\left(\mathrm{Rp}_{\text {ióvenes }}=193.1 ; \mathrm{RP}_{\text {adultos }}\right.$ $\left.=210.87, \mathrm{PS}_{\mathrm{est}}=.45\right)$.

Por otra parte, en la dimensión realización personal, se observan diferencias a favor de las jóvenes en el ítem 21 (Estoy satisfecho con lo que hasta ahora he alcanzado) $\left(\mathrm{Rp}_{\text {jóvenes }}=203.78 ; \mathrm{Rp}_{\text {adultos }}=198.96, \mathrm{PS}_{\text {est }}=\right.$ .48) y el ítem 27 (Creo que no me falta nada) ( $R p_{\text {jóvenes }}$ $\left.=207.16 ; \mathrm{Rp}_{\text {adultos }}=195.18, \mathrm{PS}_{\text {est }}=.47\right)$. Además, la diferencia más pequeña a favor de los adultos se da en el ítem 8 (Hasta ahora he conseguido las cosas que para mí son importantes) $\left(\mathrm{Rp}_{\text {jóvenes }}=191.56\right.$; $\mathrm{Rp}_{\text {adultos }}$ $\left.=212.56, \mathrm{PS}_{\text {est }}=.44\right)$. Finalmente, en la dimensión alegría de vivir, se observan diferencias cercanas a ser consideradas pequeñas a favor de los adultos en el ítem 12 (Es maravilloso vivir) $\left(\mathrm{Rp}_{\text {jóvenes }}=194.08 ; \mathrm{Rp}_{\text {adultos }}\right.$ $\left.=209.78, \mathrm{PS}_{\text {est }}=.46\right)$ y la diferencia más pequeña se encuentra en el ítem 15 (Soy una persona optimista) $\left(\mathrm{Rp}_{\text {jóvenes }}=185.52 ; \mathrm{Rp}_{\text {adultos }}=219.33, \mathrm{PS}_{\text {est }}=.41\right)$.

\section{Discusión}

El estudio tuvo como objetivo la comparación de la felicidad de acuerdo con el sexo y edad. Los resultados evidencian que las mujeres, comparadas con los varones, son más propensas a experimentar cierto grado de felicidad, lo cual concuerda con estudios previos (Francis, 1999; Lu, 1996; Mookherjee, 1997; Pavot, Diener, et al, 1990; Seligman, 2011; Wood, Rhodes et al, 1989).

En relación con estos hallazgos, estudios reportan que las mujeres evalúan como menos compleja, incierta y más interesante y emocionante la expresión de conductas positivas (Kashdan, Mishra, Breen \& Froh, 2009). Asimismo, las mujeres con una mayor 
expresión de conductas positivas, son más propensas, satisfacen una serie de necesidades psicológicas como la pertenencia y autonomía. Un resultado importante está en relación a que las mujeres tienden a puntuar más alto en conductas asociadas al optimismo y la satisfacción con su vida, lo que concuerda con estudios anteriores, donde las mujeres tienden a ser más optimistas y satisfechas consigo mismas (Salgado, 2009). Los resultados podrían estar explicados también por la influencia de pautas culturales en la expresión de emociones, las cuales se transmiten de generación en generación. En este sentido, los roles culturales ejercidos por hombres y mujeres, tendrían un papel importante para la felicidad en la muestra estudiada (Alarcón \& Caycho, 2015).

Desde una perspectiva psicobiológica, ciertas características diferenciales universales de las mujeres, como una mayor sensibilidad y vulnerabilidad relacionadas con el estrógeno y la producción de progesterona, podrían brindar algún tipo de explicación (Lippa, 2005). Así, también, se encuentra evidencia de que mayores niveles de cortisol y menores niveles de hemoglobina glicosilada (Lindfors \& Lundberg, 2002; Ryff, Singer \& Love, 2004) están en relación con mayores niveles de experiencias positivas como la felicidad. A pesar de la evidencia, algunos autores (Nydegger, 2004) señalan también que las explicaciones biológicas para estos resultados diferenciados no encuentran un adecuado apoyo empírico.

Por otro lado, es importante tener en consideración, en las diferencias de género de la felicidad, la participación de factores asociados a las condiciones de vida de hombres y mujeres (Easterlin, 2002; Russo \& Green 1993). En este sentido, el grado de desigualdad de género puede tener alguna influencia en las diferencias de felicidad entre hombres y mujeres, las cuales deben ser menores en sociedades con igualdad de oportunidades en comparación con sociedades donde las oportunidades son menores para las mujeres (Tesch-Romer, Motel-Klingebiel \& Tomasik, 2008). El Perú, en el 2015, ocupó el puesto 89 dentro del ranking mundial de equidad de género dentro de un total de 145 países, retrocediendo 44 puestos en comparación con el año 2014 (World Economic Forum, 2015). De acuerdo con la hipótesis de la desigualdad de género de las sociedades, esta situación podría explicar la ausencia de efecto de las diferencias entre hombres y mujeres en relación a la felicidad.

Los resultados, en función al sexo, reportados en el presente estudio no concuerdan con otras investigaciones previas que no encontraron diferencias significativas (Argyle, 1990; Diener \& Diswas, 2008; Laca, Verdugo et al., 2005; Seligman, 2011). En un intento por explicar esto, Diener et al. (1999), señalan que las mujeres pueden experimentar más intensamente y al mismo tiempo emociones positivas y negativas, lo que generaría un equilibrio entre ambos afectos. Lo anterior sugeriría que nuestros resultados se encuentran limitados al grupo de edad estudiado.

En relación con la edad, se observa que, en promedio, las personas de mayor edad tienen más altas puntuaciones en la EFL que las de menor edad. En esta línea, Romero (2015), señala que mientras más edad se tiene, las expresiones de conductas positivas contribuyen a mejorar las relaciones personales, además de servir como factor de protección ante diversos problemas. Por otro lado, Moyano y Ramos (2007), mencionan que las personas más jóvenes tienden a disfrutar en menor medida el presente que las personas mayores.

La anterior se explica debido a que a mayor edad las personas se sienten más valoradas socialmente, permitiendo orientar los pensamientos hacia situaciones de carácter más positivo. Asimismo, diversos autores (Alarcón, 2001; Carstensen, 1991, 1995; Labovic-Vief \& Blanchard-Fields, 1982), señalan que a mayor edad, las personas regulan mejor sus emociones, maximizando los afectos positivos y a minimizando los negativos.

Los resultados anteriores, en relación con el sexo y la edad son importantes pues algunos estudios (Inglehart, 2002). Las posibles diferencias en las conductas positivas pueden ser explicadas también gracias a la interacción entre el sexo y la percepción de bienestar. 
En el presente estudio, dado su carácter exploratorio, se debe reconocer una serie de limitaciones inherentes al tamaño y representatividad de la muestra, por lo que los resultados deben interpretarse con precaución. El empleo de un muestreo por conveniencia disminuye la capacidad de generalización de los resultados, por lo que, próximos estudios deberían contar con un número mayor de participantes provenientes de diferentes contextos sociales, permitiendo así la replicabilidad de los resultados aquí descritos. Asimismo, al utilizar una muestra de jóvenes y adultos, no se tiene total claridad de si los resultados podrían generalizarse a otros grupos de edad o para las mismas personas a medida que envejecen. Además, el utilizar un diseño transversal impide establecer relaciones causales entre la felicidad y las variables sociodemográficas estudiadas. Finalmente, el estudio se realizó con base en medidas de autoinforme, lo que sugiere que, métodos multicéntricos podrían mejorar la robustez de los hallazgos (Diener, 1994).

Una de las principales contribuciones del estudio radica en que es uno de los pocos, en el contexto peruano, que evalúan la felicidad y su relación con variables sociodemográficas desde una perspectiva psicológica. En conclusión, la existencia de diferencias significativas de la felicidad entre hombres y mujeres es de suma importancia tanto en la investigación como intervención en contextos clínicos y sociales.

\section{Referencias}

Alarcón, R. (2001). Relaciones entre felicidad, género, edad y estado conyugal. Revista de Psicología, 19(1), $27-46$.

Alarcón, R. (2006). Desarrollo de una escala factorial para medir la felicidad. Revista Interamericana de Psicología, 40(1), 99-106.

Alarcón, R. (2009). Psicología de la felicidad. Introducción a la psicología positiva. Lima: Universidad Ricardo Palma.

Alarcón, R., \& Caycho, T. (2015). Relaciones entre gratitud y felicidad en estudiantes universitarios de Lima Metropolitana. Psychologia: avances de la disciplina, 9(1), 59-69.
Argyle, M. (1990). Relaciones Sociales. En M. Hewstone, W. Stroebe, J. P. Codol y Stephenson, G. M. (Eds.) Introducción a la Psicología Social. Una perspectiva europea. (pág. 222-244) (J. F. Morales, Trad.). Barcelona, España: Ariel (Trabajo original publicado en 1988).

Bailey, T., Eng, W., Frisch, M., \& Snyder, C. (2007). Hope and optimism as related to life satisfaction. The Journal of Positive Psychology, 2(3), 168-175. doi: 10.1080/17439760701409546

Blanchflower, D. G., \& Andrew, J. O. (2000). Well-being over Time in Britain and the USA. National Burean of Economic Research Working PaperNo. 7487. Recuperado de http://www.nber.org/papers/w7487

Bono, G., Emmons, R. A., \& McCullough, M. E. (2004). Gratitude in practice and the practice of gratitude. En P. A. Linley y S. Joseph (Eds.), Positive psychology in practice, (pp. 464-481). Hoboken, NJ: John Wiley, \& Sons, Inc.

Carstensen, L. L. (1991). Selective theory: Social activity in life-span contex. Annual Review of Gerontology and Geriatrics, 11,195-217.

Carstensen, L. L. (1995). Evidence for life-span theory of socioemotional selective. Current Directions in Psychologilcal Science, 4(5), 151-156.

Caycho, T. (2010). Variables psicológicas asociadas con la felicidad en centros peri-urbanos y urbanomarginales de Lima. Scientia UCV, 2(1), 61-68.

Costa, P. T., Zonderman, A. B., McCrae, R. R., Huntley, J. C., Locke, B. Z., \& Barbano, H. E. (1987). Longitudinal analyses of psychological well-being in a national sample: Stability of mean levels. Journal of Gerontology, 42(1), 50-55.

Cuadra, H., \& Florenzano, R. (2003). La psicología positiva: un cambio a nuestro enfoque patológico clásico. Liberabit, 10, 82-88.

Chen, H., \& Chen, J. (2008). Estructural equation modeling: Hope, coping styles and emotions of impoverished undergraduates. Chinese Journal of Clinical Psychology. 16(4), 392-394. 
Csikszentmihalyi, M. (2008). El yo evolutivo. Una psicología para un mundo globalizado. Barcelona: Kairos.

Diener, E. (1984). Subjective well-being. Psychological Bulletin, 95(3), 542-575.

Diener, E., Emmons, R., Larsen, R., \& Griffin, S. (1985). The satisfaction with life scale. Journal of personality assessment, 49(1), 71-75. doi: 10.1207/ s15327752jpa4901_13

Diener, E., \& Suh, M. E. (1998). Subjective well-being and age: An international analysis. En S. K. Wamer y L. M. Powell (Eds.), Annual review of gerontology and geriatric (pp. 304-324). Nueva York: Spinger Publishing.

Diener, E. (1994). Assessing subjective well-being: Progress and opportunities. Social Indicators Research, 31(2), 103-157. doi: 10.1007/BF01207052

Diener, E., Suh, E., Lucas, R., \& Smith, H. (1999). Subjective well-being: three decades of progress. Psychological Bulletin, 125(2), 271-301. doi: 10.1037//0033-2909.125.2.276

Diener, E., \& Diswas, R. (2008). Happiness. Unlocking the Mysteries of psychological wealth. E. U. A.: Blackwell Publishing.

Easterlin, R. A. (2003). Happiness of Women and Men in Later Life: Nature, Determinants, and Prospects. En M. J. Sirgy, D. Rahtz y J. Samli (Eds.). Advances in Quality-of-Life Theory and Research. (pp. 13-26). Netherlands: Kluwer Academic Publishers.

Emmons, R. A., \& McCullough, M. E. (2003). Counting blessings versus burdens: An experimental investigation of gratitude and subjective well-being in daily life. Journal of Personality and Social Psychology, 84(2), 377-89. doi: 10.1037/0022-3514.84.2.377

Emmons, R. A. (2008). Gratitude, subjective wellbeing, and the brain. En M. Eid \& R. J. Larsen (Eds.), The Science of Subjective Wellbeing (pp. 469489). New York: Guilford Press.

Erceg-Hurn, D., \& Mirosevich, V. (2008). Modern robust statistical methods: An easy way to maximize the accuracy and power of your research. American Psychologist, 63(7), 591-601. doi: 10.1037/0003-066X.63.7.591

Francis, L. J. (1999). Happiness is a thing called stable extraversion: a further examination of relationship between the Oxford Happiness Inventory and Eysenck's dimensional model of personality and gender. Personality and Individual Differences, 26(1), 5-11. doi: 10.1016/S0191-8869(98)00185-8

Frey, B. S., \& Stutzer, A. (2000) Happiness, Economy and Institutions. Economic Journal, 110(446), 918-38. doi: 10.1111/1468-0297.00570

Grissom, R. J. (1994). Probability of the superior outcome of one treatment over another. Journal of Applied Psychology, 79(2), 314 -316. doi: 10.1037/0021-9010.79.2.314

Halama, P. (2010). Hope as a mediator between personality traits and life satisfaction. Studia Psychologica, 52(4), 309-314.

Hernández, R., Fernández, C., \& Baptista, P. (2010). Metodología de la investigación. México: Mc Graw Hill.

Inglehart, R. (2002). Gender, aging, and subjective wellbeing. InternationalJournalof Comparative Sociology, 43(35), 391-408. doi: 10.1177/002071520204300309

Kashdan, T. B., Mishra, A., Breen, W. E., \& Froh, J. J. (2009). Gender differences in gratitude: Examining appraisals, narratives, the willingness to express emotions, and changes in psychological needs. Journal of Personality, 77(3), 691-730. doi: 10.1111/j.1467-6494.2009.00562.x

Labouvie-Vief, G., \& Blanchard-Fields, F. (1982). Cognitive ageing and psychological growth. Ageing and Society, 2(2), 183-209. doi: 10.1017/ S0144686X00009429

Laca, F., Verdugo, J., \& Guzman, M. (2005). Satisfacción con la vida de algunos colectivos mexicanos: una discusión sobre la psicología del bienestar subjetivo. Enseñanza en Investigación en Psicología, 10(2), 325-336.

Lindfors, P., \& Lundberg, U. (2002). Is low cortisol release an indicator of positive health?. Stress and Health, 18(4), 153-160. doi: 10.1002/smi.942 
Lippa, R. (2005). Gender, nature, and nurture (2 ed.). Mahwah, NJ: Lawrence Erlbaum

Lu, L. (1996). Correlates of happiness among the Chinesepeople. Taipei, Taiwan, ROC: National Science Council.

Lyubomirsky, S. (2008). La ciencia de la felicidad. Un método para conseguir el bienestar. España: Urano.

Macnulty, W. K. (2004). Self-schemas, forgiveness, gratitude, physical health, and subjective well being. Dissertation Abstracts International, 65, 2683B.

Makinen, J. A., \& Jhonson, S. M. (2006). Resolving attachment injuries in couples using emotionally focused therapy: Step toward forgiveness and reconciliation. Journal of Consulting and Clinical Psychology, 74(6), 1055-1064. doi: 10.1037/0022006X.74.6.1055

Michalos, A. C. (1995a). Tecnología y calidad de vida. Intervención Psicosocial, 4(10), 51-55.

Michalos, A. C. (1995b). Introducción a la teoría de las discrepancias múltiples [TDM]. Intervención Psicosocial, 4(12), 99-115.

Montero, O., \& León, I. (2007). A guide for naming studies in psychology. International Journal of Clinical and Health Psychology, 7(3), 847-862.

Mookherjee, H. N. (1997). Marital status and perception of well-being. The Journal of Social Psychology, 137(1), 95-105.

Moyano, E., \& Ramos, N. (2007). Bienestar subjetivo: midiendo satisfacción vital, felicidad y salud en población chilena de la Región Maule. Universum, 22(2), 177-193.

Mroczek, D. K., \& Kolarz, C. M. (1998). The effect of age on positive and negative affect: A developmental perspective on happiness. Journal Personality and Social Psychology, 75(5), 1333-1349. doi: 10.1037/0022-3514.75.5.1333

Myers, D. G. (2000). The funds, friends, and faith of happy people. American Psychologist, 55(1), 56-67. doi: 10.1037/0003-066X.55.1.56
Myers, D. G., \& Diener, E. (1996). The pursuit of happiness. Scientific American, 274(5), 70-72.

Nydegger, R. (2004). Gender and mental health: Incidence and treatment issues. En M. A. Paludi (Ed.), Praeger guide to the psychology of gender (pp. 93116). Westport: Praeger/Greenwood.

Niven, D. (2011). Los 100 secretos de la gente feliz: Colombia: Norma.

Pavot, W., Diener, E., \& Fujita, F. (1990). Extraversion and happiness. Personality and Individual Differences, 11(12), 1299-1306. doi: 10.1016/01918869(90)90157-M

Romero, M. V. (2015). La gratitud como fortaleza humana: una revisión bibliográfica. Recuperado de http://tauja.ujaen.es/bitstream/10953.1/1982/1/ Romero_Gonzlez_MVictoria_TFG_Psicologa.pdf

Russo, N. F., \& Green, B. L. (1993). Women and mental health. En F. L. Denmakr \& M. A. Paludi (Eds.), Psychology of women: $A$ bandbook of issues and theories (pp. 379-436). Westport, CT: Greenwood.

Ryff, C. D., Singer, B. H., \& Love, G. D. (2004). Positive health: Connecting well-being with biology. Philosophical Transactions-Royal Society of London Series B Biological Sciences, 359, 1383-1394.

Salgado, A. C. (2009). Felicidad, resiliencia y optimismo en estudiantes de colegios nacionales de la Ciudad de Lima. Liberabit, 2(15), 133-141.

Seligman, M. E. P. \& Csikszentmihalyi, M. (2000). Positive psychology: an introduction. American Psychologist, 55(1), 5-14.

Seligman, M. (2006). La auténtica felicidad. Buenos Aires: Vergara.

Seligman, M. (2011). La auténtica felicidad. España: Byblos.

Tesch-Römer, C., Motel-Klingebiel, A., \& Tomasik, M. J. (2008). Gender differences in subjective wellbeing: Comparing societies with respect to gender equality. Social Indicators Research, 85(2), 329-349. doi: 10.1007/s11205-007-9133-3 
Veenhoven, R. (2005). Lo que sabemos de la felicidad. En E. L. Garduño., A. B. Salinas y H. M. Rojas (Eds.), Calidad de vida y bienestar subjetivo en México (pp. 17-55). México: Plaza y Valdés.

Wood, W., Rhoes, N., \& Whelan, M. (1989). Sex differences in positive well-being: a consideration of emotional style and marital status. Psychological Bulletin, 106(2), 249-264. doi: 10.1037/00332909.106.2.249

World Economic Forum (2015). The Global Gender Gap Report 2015. Disponible en http://www3.weforum. org/docs/GGGR2015/cover.pdf

Recibido: 19 de mayo de 2016 Aceptado: 19 de octubre de 2016 
